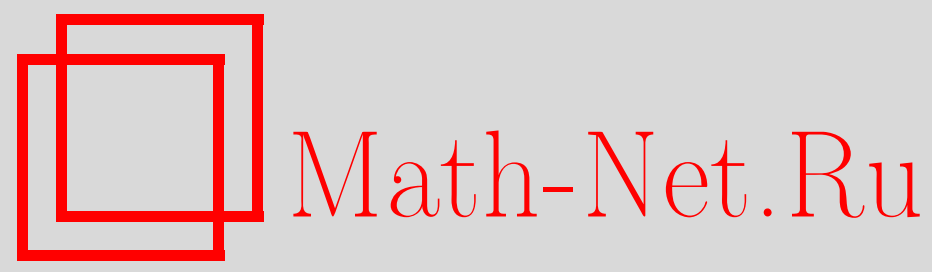

М. И. Черданцев, Асимптотика собственного значения оператора Лапласа в области с сингулярно возмущенной границей, Матем. заметки, 2005, том 78, выпуск 2, 299-307

DOI: https://doi.org/10.4213/mzm2575

Использование Общероссийского математического портала Math-Net.Ru подразумевает, что вы прочитали и согласны с пользовательским соглашением http://www.mathnet.ru/rus/agreement

Параметры загрузки:

IP: 54.205 .225 .156

26 апреля 2023 г., 16:06:38

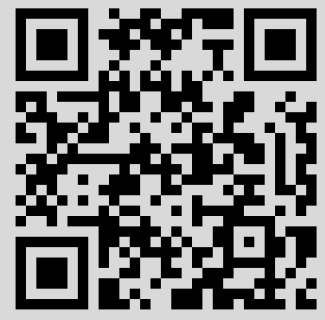




\section{АСИМПТОТИКА СОБСТВЕННОГО ЗНАЧЕНИЯ ОПЕРАТОРА ЛАПЛАСА В ОБЛАСТИ С СИНГУЛЯРНО ВОЗМУЩЕННОЙ ГРАНИЦЕЙ}

\section{М. И. Черданцев}

В работе рассматривается задача на собственные значения оператора Лапласа в трехмерной области с сингулярно возмущенной границей. Возмущение осуществляется заданием дополнительного граничного условия Дирихле на малой незамкнутой поверхности находящейся внутри области. Исследуется сходимость и асимптотическое поведение простых собственных значений задачи.

Библиография: 10 названий.

1. Постановка задачи и формулировка результата. Известно (см., например, [1]), что волновая функция, описывающая состояние свободной частицы, удовлетворяет стационарному уравнению Шрёдингера

$$
\frac{\hbar^{2}}{2 m} \Delta \psi+E \psi=0,
$$

где $\hbar$ - постоянная Планка, $m$ - масса частицы, а $E$ - энергия частищы. В случае, когда частица находится в ограниченной области, ее волновая функция на границе этой области обращается в нуль. Если внутри области имеется некоторое препятствие, то на нем волновая функция также обращается в нуль. Данная задача соответствует краевой задаче на собственные значения оператора Лапласа с граничным условием Дирихле. В настоящей работе исследуется поведение собственных значений задачи в случае, когда область является трехмерной, а препятствие имеет нулевую толщину и малые линейные размеры: $\gamma_{\varepsilon}=\left\{x: x \varepsilon^{-1} \in \gamma\right\}$, где $\gamma$-поверхность, диффеоморфная открытому кругу и содержащая начало координат, а $0<\varepsilon \ll 1-$ мальй параметр. При $\varepsilon \rightarrow 0$ поверхность $\gamma_{\varepsilon}$ стягивается к началу координат. Пусть $\Omega \subset \mathbb{R}^{3}$ - односвязная ограниченная область, содержащая начало координат, с границей $\Gamma \in C^{\infty}$. Обозначим $\Omega_{\varepsilon}=\Omega \backslash \overline{\gamma_{\varepsilon}}, \Gamma_{\varepsilon}=\Gamma \cup \gamma_{\varepsilon}$. Рассматривается следующая краевая задача на собственные значения:

$$
-\Delta \psi_{\varepsilon}=\lambda_{\varepsilon} \psi_{\varepsilon} \quad \text { при } x \in \Omega_{\varepsilon}, \quad \psi_{\varepsilon}=0 \text { при } x \in \Gamma_{\varepsilon},
$$

которую мы будем называть возмущенной. Здесь $\lambda_{\varepsilon}=\left(2 m / \hbar^{2}\right) E$. Задачу

$$
-\Delta \psi_{0}=\lambda_{0} \psi_{0} \quad \text { при } x \in \Omega, \quad \psi_{0}=0 \text { при } x \in \Gamma,
$$

будем назьвать предельной.

Основной результат данной работы сформулируем в следующем утверждении.

Работа выполнена при поддержке Российского фонда фундаментальных исследований, программы "Ведущие научные школы" и программы "Университеты России". 
Теорема 1.1. Если $\lambda_{0}$ - простое собственное значение предельной задачи, то существует единственное и простое собственное значение $\lambda_{\varepsilon}$ возмущенной задачи, сходящееся при $\varepsilon \rightarrow 0 \kappa \lambda_{0}$, а его асимптотика имеет вид

$$
\begin{gathered}
\lambda_{\varepsilon}=\lambda_{0}+\varepsilon \lambda_{1}+o(\varepsilon) \quad n p u \quad \varepsilon \rightarrow 0, \\
\lambda_{1}=4 \pi \psi_{0}^{2}(0) c(\gamma) .
\end{gathered}
$$

Здесь $\psi_{0}$ - нормированная в $L_{2}(\Omega)$ собственная функиия, соответствующая собственному значению $\lambda_{0}$, a $c(\gamma)>0$ - некоторая константа, зависящая от геометрии поверхности $\gamma$, которая будет определена ниже.

Данное возмущение носит сингулярный характер. Поэтому при построении асимптотики используется метод согласования асимптотических разложений [2]. Заметим, что асимптотика собственных значений в области с малым отверстием строилась в [3].

2. Сходимость собственного значения возмущенной задачи. В данном пункте будет доказана сходимость собственных значений и собственных функций возмущенной задачи к соответствующим собственным значениям и собственным функциям предельной задачи. Используемая схема доказательства аналогична примененной в [4]-[7] для других типов сингулярных возмущений краевых задач.

Будем использовать следующие обозначения: $L_{2}(G)$ и $W_{2}^{1}(G)$ - пространства Соболева с нормами $\|\cdot\|_{0, G},\|\cdot\|_{1, G}$ и скалярными произведениями $(\cdot, \cdot)_{0, G},(\cdot, \cdot)_{1, G}$ соответственно. Замыкание в норме $W_{2}^{1}(G)$ функций из $C^{\infty}(\bar{G})$, обращающихся в нуль в окрестности множества $g$, обозначим $W_{2,0}^{1}(G, g)$. Рассмотрим следующие краевые задачи:

$$
\begin{array}{llll}
-\Delta u_{0}=\lambda u_{0}+f & \text { в } \Omega, & u_{0}=0 & \text { на } \Gamma, \\
-\Delta u_{\varepsilon}=\lambda u_{\varepsilon}+f & \text { в } \Omega_{\varepsilon}, & u_{0}=0 & \text { на } \Gamma_{\varepsilon} .
\end{array}
$$

Решения краевых задач будем понимать в обобщенном смысле (см. [8], [9]). То есть, пусть $f \in L_{2}(\Omega)$. Решением задачи (2.1) будем называть элемент $u_{0} \in W_{2,0}^{1}(\Omega, \Gamma)$, удовлетворяющий равенству

$$
\left(\nabla u_{0}, \nabla \eta\right)_{0, \Omega}=\lambda\left(u_{0}, \eta\right)_{0, \Omega}+(f, \eta)_{0, \Omega}
$$

при любом $\eta \in W_{2,0}^{1}(\Omega, \Gamma)$. Учитьвая, что $W_{2,0}^{1}\left(\Omega_{\varepsilon}, \Gamma_{\varepsilon}\right) \subset W_{2}^{1}(\Omega)$, решением задачи $(2.2)$ назовем элемент $u_{\varepsilon} \in W_{2,0}^{1}\left(\Omega_{\varepsilon}, \Gamma_{\varepsilon}\right)$, удовлетворяюший равенству

$$
\left(\nabla u_{\varepsilon}, \nabla \eta\right)_{0, \Omega}=\lambda\left(u_{\varepsilon}, \eta\right)_{0, \Omega}+(f, \eta)_{0, \Omega}
$$

при любом $\eta \in W_{2,0}^{1}\left(\Omega_{\varepsilon}, \Gamma_{\varepsilon}\right)$.

ЛЕмма 2.1. Пусть $K$ - компакт в комплексной плоскости, не содерхащий собственных значений предельной задачи (1.2). Тогда

а) существует $\varepsilon_{0}>0$ такое, что при любом $\varepsilon<\varepsilon_{0}$ и любом $\lambda \in K$ существует единственное решение $u_{\varepsilon}$ задачи (2.2) и справедлива равномерная по $\varepsilon$ и оиенка

$$
\left\|u_{\varepsilon}\right\|_{1, \Omega} \leqslant C\|f\|_{0, \Omega}
$$

б) имеет место сходимость

$$
u_{\varepsilon} \rightarrow u_{0} \quad \text { сильно в } W_{2}^{1}(\Omega) \quad \text { при } \varepsilon \rightarrow 0 .
$$


ДокАЗАТЕЛЬство. Известно (см., например, [9]), что для доказательства существования единственного решения задачи (2.2) достаточно показать справедливость оценки (2.4). Допустим противное, т.е. существует такая последовательность $\varepsilon_{k} \rightarrow 0$ при $k \rightarrow 0$, и такие последовательности $f_{k}, \lambda_{k}$, что для решений задачи $(2.2)$ при $\varepsilon=\varepsilon_{k}$, $f=f_{k}, \lambda=\lambda_{k}$ имеет место неравенство

$$
\left\|u_{\varepsilon_{k}}\right\|_{1, \Omega} \geqslant k\left\|f_{k}\right\|_{0, \Omega} .
$$

Не ограничивая общности, можно считать, что $\left\|u_{\varepsilon_{k}}\right\|_{0, \Omega}=1$. Заменяя в (2.3) $\eta$ на $u_{\varepsilon}$ и прибавляя $\left(u_{\varepsilon}, u_{\varepsilon}\right)_{0, \Omega}$ к обеим частям равенства, легко получить априорную оценку

$$
\left\|u_{\varepsilon}\right\|_{1, \Omega} \leqslant(1+|\lambda|)\left(\|f\|_{0, \Omega}+\left\|u_{\varepsilon}\right\|_{0, \Omega}\right) .
$$

Тогда, подставив $(2.6)$ в $(2.7)$, получим

$$
\left\|u_{\varepsilon_{k}}\right\|_{1, \Omega} \leqslant C .
$$

Заметим, что из (2.8) и (2.6) следует сходимость

$$
\left\|f_{k}\right\|_{0, \Omega} \rightarrow 0 \text {. }
$$

Из (2.8) следует, что множество $u_{\varepsilon_{k}}$ ограниченно в $W_{2}^{1}(\Omega)$, а значит, слабо компактно в $W_{2}^{1}(\Omega)$ и компактно в $L_{2}(\Omega)$. Следовательно, существует подпоследовательность $\varepsilon_{k_{m}}$ такая, что

$$
\begin{gathered}
\lambda_{k_{m}} \rightarrow \lambda_{*} \in K, \\
u_{\varepsilon_{k_{m}}} \rightarrow u_{*} \neq 0 \quad \text { сильно в } L_{2}(\Omega) \text { и слабо в } W_{2}^{1}(\Omega) \text { при } m \rightarrow \infty .
\end{gathered}
$$

Пусть $\eta$ - произвольная функщия из $W_{2,0}^{1}(\Omega, \Gamma)$, обозначим $\eta_{\varepsilon}=\eta\left(1-\chi\left(|x| \varepsilon^{-1}\right)\right)$, где $\chi(t)$ - срезающая функция из класса $C^{\infty}$, равная единице при $|t|<1$ и нулю при $|t|>2$. Не ограничивая обшности, можно считать, что поверхность $\bar{\gamma}$ содержится в шаре единичного радиуса. Тогда легко видеть, что $\eta_{\varepsilon} \in W_{2,0}^{1}\left(\Omega, \Gamma_{\varepsilon}\right)$ и $\eta_{\varepsilon} \rightarrow \eta$ в норме $W_{2}^{1}(\Omega)$ при $\varepsilon \rightarrow 0$. Переходя в равенстве $(2.3)$ при $\eta=\eta_{\varepsilon}, \varepsilon=\varepsilon_{k_{m}}, \lambda=\lambda_{k_{m}}$ и $f=f_{k_{m}}$ к пределу при $m \rightarrow \infty$ и учитьвая $(2.9),(2.10)$ и $(2.11)$, получаем

$$
\left(\nabla u_{*}, \nabla \eta\right)_{0, \Omega}=\lambda_{*}\left(u_{*}, \eta\right)_{0, \Omega} .
$$

Так как $u_{*} \neq 0$, то $\lambda_{*}$ является собственньм значением предельной задачи - получили противоречие. Таким образом, оценка (2.4) верна и в силу альтернативы Фредгольма существует единственное решение задачи (2.2).

Теперь покажем, что при любом фиксированном $\lambda \in K$ имеет место сходимость (2.5). Пусть $\varepsilon_{k}-$ произвольная последовательность, сходящаяся к нулю при $k \rightarrow \infty$. Из (2.4), слабой компактности ограниченного множества в $W_{2}^{1}(\Omega)$ и компактности вложения $W_{2}^{1}(\Omega)$ в $L_{2}(\Omega)$ получаем, что существует подпоследовательность $\varepsilon_{k_{m}}$ такая, что при $m \rightarrow \infty$ справедливы сходимости (2.11). Далее, вновь переходя к пределу в (2.3) при $\eta=\eta_{\varepsilon}, \varepsilon=\varepsilon_{k_{m}}$ в силу единственности решения предельной задачи получаем, что $u_{*}=u_{0}$. Подставляя в $(2.3) \eta=u_{\varepsilon_{k_{m}}}$ и учитьвая сходимость в $L_{2}(\Omega)$, получаем

$$
\left\|u_{\varepsilon_{k_{m}}}\right\|_{1, \Omega} \rightarrow\left\|u_{0}\right\|_{1, \Omega}
$$

Отсюда и из слабой сходимости $u_{\varepsilon_{m}}$ в норме $W_{2}^{1}(\Omega)$ следует сильная сходимость в этой норме. В силу произвола в выборе последовательности $\varepsilon_{n}$ получаем, что сходимость (2.5) имеет место. Лемма доказана.

Пользуясь леммой, докажем сходимость собственных значений возмущенной задачи. 
Теорема 2.1. Пусть $\lambda_{0}$ - простое собственное значение предельной задачи (1.2), тогда к нему сходится единственное и простое собственное значение $\lambda_{\varepsilon}$ возмущенной задачи (1.1), а соответствующая ему собственная функиия $\psi_{\varepsilon}$ сходится $\kappa \psi_{0}$ сильно в $W_{2}^{1}(\Omega)$.

ДокАЗАТЕЛЬСТво. Пусть последовательность $\lambda_{0}, \lambda_{1}, \ldots, \lambda_{n}, \ldots$ содержит все собственные значения задачи (1.2), причем каждое собственное значение повторяется столько раз, какова его кратность. Существует последовательность соответствующих ортонормированных в $L_{2}(\Omega)$ собственных функций $\psi_{0}, \psi_{1}, \ldots, \psi_{n}, \ldots$, при этом функции

$$
\frac{\psi_{n}}{\sqrt{1+\lambda_{n}}}, \quad n=0,1, \ldots
$$

ортонормированы в $W_{2}^{1}(\Omega)$ (см., например, [9]). Любая функция из $W_{2}^{1}(\Omega)$, в частности $u_{0}$, раскладьвается в ряд Фурье по ортонормированному базису $(2.12)$ :

$$
u_{0}=\sum_{n=0}^{\infty}\left(u_{0}, \psi_{n}\right)_{1, \Omega} \frac{\psi_{n}}{1+\lambda_{n}}
$$

Подставив это разложение в правую часть равенства

$$
\left(u_{0}, \psi_{n}\right)_{1, \Omega}=(1+\lambda)\left(u_{0}, \psi_{n}\right)_{0, \Omega}+\left(f, \psi_{n}\right)_{0, \Omega}
$$

легко получить следующую формулу:

$$
u_{0}=\sum_{n=0}^{\infty} \frac{\left(f, \psi_{n}\right)_{0, \Omega}}{\lambda_{n}-\lambda} \psi_{n}
$$

Аналогично,

$$
u_{\varepsilon}=\sum_{n=0}^{\infty} \frac{\left(f, \psi_{\varepsilon, n}\right)_{0, \Omega}}{\lambda_{\varepsilon, n}-\lambda} \psi_{\varepsilon, n}
$$

где $\lambda_{\varepsilon, n}$ и $\psi_{\varepsilon, n}-$ собственные значения и ортонормированныев $L_{2}(\Omega)$ собственные функции задачи (1.1).

Обозначим через $S(t, z)$ открытьй круг на комплексной плоскости радиуса $t$ с центром в точке $z$. Так как множество собственных значений предельной задачи не имеет конечных точек накопления (см., например, [9]), то существует такое $T>0$, что $\overline{S\left(t, \lambda_{0}\right)}$ при $t<T$ не содержит собственных значений предельной задачи кроме $\lambda_{0}$. Следовательно, в силу леммы для достаточно малых $\varepsilon$ при $\lambda \in \partial S\left(t, \lambda_{0}\right)$ задача $(2.2)$ имеет единственное решение. Из (2.5) и теоремы Лебега вытекает, что при $\varepsilon \rightarrow 0$

$$
\int_{\partial S\left(t, \lambda_{0}\right)}\left\|u_{\varepsilon}(x, \lambda)-u_{0}(x, \lambda)\right\|_{1, \Omega} d \lambda \rightarrow 0
$$

Отсюда следует, что

$$
\int_{\partial S\left(t, \lambda_{0}\right)} u_{\varepsilon}(x, \lambda) d \lambda \rightarrow \int_{\partial S\left(t, \lambda_{0}\right)} u_{0}(x, \lambda) d \lambda \quad \text { в норме } W_{2}^{1}(\Omega) .
$$


Из комплексного анализа известно, что интеграл по замкнутому контуру равен сумме вычетов функции, умноженной на $2 \pi i$. Возьмем $f=\psi_{0}$, учитывая $(2.13)$ получим, что

$$
\int_{\partial S\left(t, \lambda_{0}\right)} u_{0}(x, \lambda) d \lambda=2 \pi i \psi_{0}
$$

Из (2.14), (2.15) и последнего равенства вытекает, что при достаточно малых $\varepsilon$ интеграл $\int_{\partial S\left(t, \lambda_{0}\right)} u_{\varepsilon}(x, \lambda) d \lambda$ отличен от нуля и существует собственное значение $\lambda_{\varepsilon} \in S\left(t, \lambda_{0}\right)$ возмушенной задачи, которое в силу произвола в выборе $t$ сходится к $\lambda_{0}$ при $\varepsilon \rightarrow 0$.

Доказательство сходимости $\psi_{\varepsilon} \rightarrow \psi_{0}$ в $W_{2}^{1}(\Omega)$ проводится аналогично доказательству сходимости $u_{\varepsilon} \rightarrow u_{0}$ в $W_{2}^{1}(\Omega)$ в лемме 2.1 .

Покажем, что если собственное значение $\lambda_{0}$ простое, то к нему сходится единственное и простое собственное значение $\lambda_{\varepsilon}$. Допустим, что на некоторой последовательности $\varepsilon_{n} \rightarrow 0$ существует еще одно собственное значение $\lambda_{\varepsilon_{n}}^{\prime} \rightarrow \lambda_{0}$. Соответствующая, нормированная в $L_{2}(\Omega)$, собственная функция $\psi_{\varepsilon_{n}}^{\prime}$ ортогональна $\psi_{\varepsilon_{n}}$. Но, в силу сходимости $\psi_{\varepsilon_{n}}^{\prime}$ и $\psi_{\varepsilon_{n}}$ к $\psi_{0},\left(\psi_{\varepsilon_{n}}^{\prime}, \psi_{\varepsilon_{n}}\right)_{0, \Omega} \rightarrow\left(\psi_{0}, \psi_{0}\right)_{0, \Omega}=1$ при $n \rightarrow \infty$, что противоречит ортогональности $\psi_{\varepsilon_{n}}^{\prime}$ и $\psi_{\varepsilon_{n}}$. Теорема доказана.

ЗАмЕчАниЕ. В дальнейшем, при строгом обосновании асимптотики понадобится следуюшая оценка решения задачи (2.2) при $\lambda$, близких к $\lambda_{0}$ :

$$
\left\|u_{\varepsilon}\right\|_{1, \Omega} \leqslant \frac{C}{\left|\lambda_{\varepsilon}-\lambda\right|}\|f\|_{0, \Omega},
$$

которую легко получить из (2.14).

3. Доказательство теоремы 1.1. В силу теоремы $2.1 \lambda_{\varepsilon} \rightarrow \lambda_{0}$ и $\psi_{\varepsilon} \rightarrow \psi_{0}$. Поэтому $\lambda_{0}$ и $\psi_{0}$ являются главными членами асимптотик $\lambda_{\varepsilon}$ и $\psi_{\varepsilon}$. Однако функция $\psi_{0}$ не удовлетворяет граничному условию Дирихле на $\gamma_{\varepsilon}$. Следуя методу согласования асимптотических разложений [2]-[5], [7] в малой окрестности $\gamma_{\varepsilon}$, приближение функции $\psi_{\varepsilon}$ будем искать зависящим от растянутых переменных $\xi=x \varepsilon^{-1}$. Для этого разложим функцию $\psi_{0}(x)$ в нуле в ряд Тейлора:

$$
\psi_{0}(x)=\sum_{i=0}^{\infty} P_{i}(x), \quad x \rightarrow 0,
$$

г де $P_{i}(x)$ - однородный полином степени $i$. Заметим, что $P_{0}(\xi) \equiv \psi_{0}(0)$. В переменных $\xi$ этот ряд примет вид

$$
\psi_{0}(x)=\sum_{i=0}^{\infty} \varepsilon^{i} P_{i}(\xi), \quad \varepsilon \rho=\varepsilon|\xi| \rightarrow 0 .
$$

Принимая во внимание (3.1) и следуя методу согласования асимптотических разложений, приближение функции $\psi_{\varepsilon}(x)$ в малой окрестности $\gamma_{\varepsilon}$ будем искать в виде

$$
\begin{gathered}
\psi_{\varepsilon}(x) \approx \widehat{v}_{\varepsilon}(\xi)=\sum_{i=0}^{3} \varepsilon^{i} v_{i}(\xi), \\
v_{i}(\xi) \sim P_{i}(\xi), \quad \rho \rightarrow \infty, \quad i=0,1,2,3 .
\end{gathered}
$$


Функции $v_{i}(\xi)$ будем считать определенными в $\mathbb{R}^{3} \backslash \bar{\gamma}$. Подставляя $(3.2)$ в $(1.1)$, для функций $v_{0}$ и $v_{1}$ получим краевые задачи

$$
\Delta v_{i}=0 \text { при } \xi \in \mathbb{R}^{3} \backslash \bar{\gamma}, \quad v_{i}=0 \text { при } \xi \in \gamma, \quad i=0,1 .
$$

Для обоснования асимптотики $\lambda_{\varepsilon}$ от функций $v_{2}$ и $v_{3}$ достаточно потребовать, чтобы они удовлетворяли краевьм задачам

$$
-\Delta v_{i}=\lambda_{0} P_{i-2} \quad \text { при } \xi \in \mathbb{R}^{3} \backslash \bar{\gamma}, \quad v_{i}=0 \text { при } \xi \in \gamma, \quad i=2,3 .
$$

Решение задач (3.4) и (3.5) будем искать в виде

$$
v_{i}=\hat{v}_{i}+(1-\chi(\rho)) P_{i}, \quad i=0,1,2,3 .
$$

Тогда на функции $\hat{v}_{i}, i=0,1,2,3$, получим краевые задачи

$$
-\Delta \hat{v}_{i}=f_{i} \text { при } \xi \in \mathbb{R}^{3} \backslash \bar{\gamma}, \quad \hat{v}_{i}=0 \text { при } \xi \in \gamma,
$$

где

$$
\begin{gathered}
f_{0}=-\Delta \chi \cdot P_{0}, \quad f_{1}=-\Delta \chi \cdot P_{1}-\nabla \chi \cdot \nabla P_{1}, \\
f_{i}=-\Delta \chi \cdot P_{i}-\nabla \chi \cdot \nabla P_{i}+\lambda_{0} \cdot \chi \cdot P_{i-2}, \quad i=2,3 .
\end{gathered}
$$

Аналогично тому, как это было сделано в [10, глава 16], можно показать, что, если $f-$ функция из $L_{2}\left(\mathbb{R}^{3} \backslash \bar{\gamma}\right)$ с компактным носителем, то существует единственное решение задачи

$$
-\Delta u=f \quad \text { в } \mathbb{R}^{3} \backslash \bar{\gamma}, \quad u=0 \text { на } \gamma .
$$

Отсюда следует, что задачи краевые (3.6) имеют единственные решения, причем их асимптотика на бесконечности имеет вид (см. [2])

$$
\hat{v}_{i}(\xi)=\sum_{j=0}^{\infty} \frac{X_{j}^{(i)}(\xi)}{\rho^{2 j+1}}, \quad \rho \rightarrow \infty, \quad i=0,1,2,3,
$$

где $X_{j}^{(i)}$ - однородные полиномы степени $j$. Таким образом, задачи $(3.4),(3.5)$ имеют решения с асимптотикой

$$
v_{i}(\xi)=P_{i}(\xi)+\sum_{j=0}^{\infty} \frac{X_{j}^{(i)}(\xi)}{\rho^{2 j+1}}, \quad \rho \rightarrow \infty, \quad i=0,1,2,3
$$

Покажем, что $X_{0}^{(0)}$ отличен от нуля (этот факт важен для построения асимптотики $\left.\lambda_{\varepsilon}\right)$. Пусть $B_{R}$ - открытьй шар радиуса $R$. Проинтегрируем $v_{0} \Delta v_{0}$ по частям по области $B_{R} \backslash \bar{\gamma}$ :

$$
\int_{B_{R} \backslash \bar{\gamma}} v_{0} \Delta v_{0} d \xi=-\int_{B_{R} \backslash \bar{\gamma}} \nabla v_{0} \nabla v_{0} d \xi+\int_{\gamma} v_{0} \frac{\partial v_{0}}{\partial n} d s+\int_{\rho=R} v_{0} \frac{\partial v_{0}}{\partial n} d s .
$$


Интеграл слева и второе слагаемое справа равны нулю в силу того, что функция $v_{0}$ является решением задачи (3.4). Переходя к пределу при $R \rightarrow \infty$ и подставляя вместо функции $v_{0}$ ее асимптотику, получим

$$
X_{0}^{(0)}=-\frac{1}{4 \pi \psi_{0}(0)}\left\|\nabla v_{0}\right\|_{0, R^{3} \backslash \bar{\gamma}}^{2}
$$

Рассмотрим краевую задачу

$$
\Delta V=0 \text { при } \xi \in \mathbb{R}^{3} \backslash \bar{\gamma}, \quad V=1 \text { при } \xi \in \gamma .
$$

Она имеет единственное решение в классе убывающих на бесконечности функций с асимптотикой $c(\gamma) / \rho+O\left(\rho^{-2}\right)$. Легко видеть, что $v_{0}=\psi_{0}(0)(1-V)$, поэтому предпоследнее равенство можно переписать в виде

$$
X_{0}^{(0)}=-\frac{\psi_{0}(0)}{4 \pi}\|\nabla V\|_{0, R^{3} \backslash \bar{\gamma}}^{2}=-\psi_{0}(0) c(\gamma), \quad c(\gamma)=\frac{1}{4 \pi}\|\nabla V\|_{0, R^{3} \backslash \bar{\gamma}}^{2}>0
$$

Это представление $X_{0}^{(0)}$ более наглядно, так как значение $c(\gamma)$ зависит только от вида поверхности $\gamma$.

Переписывая асимптотику $\hat{v}_{\varepsilon}(\xi)$ в переменных $x$, получаем

$$
\psi_{\varepsilon}(x) \approx \hat{v}_{\varepsilon}\left(x \varepsilon^{-1}\right)=\sum_{i=0}^{3} P_{i}(x)-\varepsilon \frac{\psi_{0}(0) c(\gamma)}{r}+O\left(\varepsilon^{2} r^{-1}\right), \quad r \varepsilon^{-1} \rightarrow \infty
$$

где $r=|x|$. Следуя методу согласования асимптотических разложений, собственную функцию вне малой окрестности $\gamma_{\varepsilon}$ и собственное значение будем искать в виде

$$
\begin{gathered}
\psi_{\varepsilon}(x) \approx \widehat{\psi}_{\varepsilon}(x)=\psi_{0}(x)+\varepsilon \psi_{1}(x), \\
\psi_{1}(x) \sim-\frac{\psi_{0}(0) c(\gamma)}{r}, \quad r \rightarrow 0, \\
\lambda_{\varepsilon} \approx \hat{\lambda}_{\varepsilon}=\lambda_{0}+\varepsilon \lambda_{1} .
\end{gathered}
$$

Подставляя (3.8) и (3.9) в (1.1), получим краевую задачу

$$
-\Delta \psi_{1}=\lambda_{0} \psi_{1}+\lambda_{1} \psi_{0} \quad \text { при } x \in \Omega \backslash\{0\}, \quad \psi_{1}=0 \text { при } x \in \Gamma \text {. }
$$

Легко видеть, что функция

$$
Y=-\psi_{0}(0) c(\gamma) \frac{\cos \left(\sqrt{\lambda_{0}} r\right)}{r}
$$

имеет асимптотику

$$
-\frac{\psi_{0}(0) c(\gamma)}{r}+O(r) \quad \text { при } \quad r \rightarrow 0
$$

и удовлетворяет уравнению

$$
-\Delta Y=\lambda_{0} Y \quad \text { при } \quad x \in \Omega \backslash\{0\} \text {. }
$$


Поэтому функцию $\psi_{1}$ будем искать в виде

$$
\psi_{1}=\chi(r) Y+\widehat{Y} .
$$

Подставив это равенство в $(3.10)$, получим

$$
-\Delta \widehat{Y}=\lambda_{0} \widehat{Y}+f+\lambda_{1} \psi_{0} \quad \text { при } x \in \Omega, \quad \widehat{Y}=0 \text { при } x \in \Gamma,
$$

где $f=\nabla Y \nabla \chi+Y \Delta \chi$. Известно, что для существования единственного решения этой краевой задачи, необходимо и достаточно вьполнения условия

$$
\left(f+\lambda_{1} \psi_{0}, \psi_{0}\right)_{\Omega}=0 .
$$

Таким образом, существует $\lambda_{1}$, а именно $\lambda_{1}=-\left(f, \psi_{0}\right)_{\Omega}$, при котором задача (3.10) имеет единственное решение с асимптотикой

$$
\psi_{1}=-\frac{\psi_{0}(0) c(\gamma)}{r}+O(r) \quad \text { при } \quad r \rightarrow 0 .
$$

Покажем справедливость формулы (1.4) для $\lambda_{1}$. Умножая уравнение (3.10) на $\psi_{0}$, интегрируя по области $\Omega \backslash B_{R}$ и применяя формулу Остроградского, получим

$$
\int_{r=R} \frac{\partial \psi_{1}}{\partial r} \psi_{0} d s+\int_{\Omega \backslash B_{R}} \nabla \psi_{1} \nabla \psi_{0} d x=\int_{\Omega \backslash B_{R}} \lambda_{0} \psi_{1} \psi_{0} d x+\int_{\Omega \backslash B_{R}} \lambda_{1} \psi_{0} \psi_{0} d x .
$$

Переходя в этом равенстве к пределу при $R \rightarrow 0$, подставляя вместо $\psi_{0}$ и $\psi_{1}$ их асимптотики и учитывая, что $\int_{\Omega} \nabla \psi_{1} \nabla \psi_{0} d x=\int_{\Omega} \lambda_{0} \psi_{1} \psi_{0} d x$, получим

$$
\lambda_{1}=4 \pi \psi_{0}^{2}(0) c(\gamma)
$$

Теперь перейдем к строгому обоснованию построенной асимптотики собственного значения.

Лемма 3.1. Для $\hat{\lambda}_{\varepsilon}$ справедлива оценка

$$
\left|\lambda_{\varepsilon}-\hat{\lambda}_{\varepsilon}\right|=o(\varepsilon)
$$

ДоКАЗАТЕЛЬСТВо. Обозначим

$$
\widetilde{\psi}_{\varepsilon}(x)=\chi\left(r \varepsilon^{-\alpha}\right) \hat{v}_{\varepsilon}\left(x \varepsilon^{-1}\right)+\left(1-\chi\left(r \varepsilon^{-\alpha}\right)\right) \widehat{\psi}_{\varepsilon}(x), \quad \text { где } \frac{2}{7}<\alpha<\frac{2}{5} .
$$

Легко видеть, что $\widetilde{\psi}_{\varepsilon} \in W_{2}^{1}(\Omega)$. Это становится очевидным, если заметить, что $\psi_{1} \in$ $W_{2}^{1}(\Omega \backslash\{0\}), v_{i}(\xi) \in W_{2}^{1}\left(B_{R} \backslash \bar{\gamma}\right)$ при любом $R, i=0,1,2,3$. Функция $\widetilde{\psi}_{\varepsilon}$ является решением краевой задачи

$$
\left(\Delta+\widehat{\lambda}_{\varepsilon}\right) \widetilde{\psi}_{\varepsilon}=\hat{f}_{\varepsilon} \quad \text { при } x \in \Omega_{\varepsilon}, \quad \widetilde{\psi}_{\varepsilon}=0 \text { на } \Gamma_{\varepsilon},
$$

где

$$
\hat{f}_{\varepsilon}=\sum_{j=1}^{3} f_{\varepsilon, j}, \quad f_{\varepsilon, 1}=\Delta \chi\left(r \varepsilon^{-\alpha}\right)\left(\hat{v}_{\varepsilon}-\widehat{\psi}_{\varepsilon}\right)+\nabla \chi\left(r \varepsilon^{-\alpha}\right) \nabla\left(\hat{v}_{\varepsilon}-\widehat{\psi}_{\varepsilon}\right),
$$




$$
\begin{aligned}
& f_{\varepsilon, 2}=\chi\left(r \varepsilon^{-\alpha}\right)\left[\Delta v_{2}+\lambda_{0} v_{0}+\varepsilon\left(\Delta v_{3}+\lambda_{0} v_{1}\right)+\varepsilon^{2} \lambda_{0} v_{2}+\varepsilon^{3} \lambda_{0} v_{3}+\varepsilon \lambda_{1} \hat{v}_{\varepsilon}\right] \\
& f_{\varepsilon, 3}=\left(1-\chi\left(r \varepsilon^{-\alpha}\right)\right)\left(\Delta \psi_{0}+\lambda_{0} \psi_{0}+\varepsilon^{-1}\left(\Delta \psi_{1}+\lambda_{0} \psi_{1}+\lambda_{1} \psi_{0}\right)+\varepsilon^{2} \lambda_{1} \psi_{1}\right) .
\end{aligned}
$$

Учитьвая асимптотики функций (3.7) и (3.11), получим, что при $\varepsilon^{\alpha}<r<2 \varepsilon^{\alpha}$ имеет место оценка $f_{\varepsilon, 1}=O\left(\varepsilon^{2-4 \alpha}+\varepsilon^{2 \alpha}\right)$. Принимая во внимание, что supp $f_{\varepsilon, 1} \subset B_{2 \varepsilon^{\alpha}} \backslash B_{\varepsilon} \alpha$, получим

$$
\left\|f_{\varepsilon, 1}\right\|_{0, \Omega}=O\left(\varepsilon^{2-5 \alpha / 2}+\varepsilon^{7 \alpha / 2}\right) .
$$

Аналогично, учитывая (3.5) и (3.10) и асимптотики функций, получаем, что

$$
\left\|f_{\varepsilon, 2}\right\|_{0, \Omega}=O\left(\varepsilon^{1+\alpha / 2}+\varepsilon^{7 \alpha / 2}\right), \quad\left\|f_{\varepsilon, 3}\right\|_{0, \Omega}=O\left(\varepsilon^{2-\alpha}\right),
$$

a $\left\|\widetilde{\psi}_{\varepsilon}\right\|_{0, \Omega}=1+o(1)$. Из этих оценок для $f_{\varepsilon, i}$ получаем, что $\left\|\hat{f}_{\varepsilon}\right\|_{0, \Omega}=o(\varepsilon)$ при $2 / 7<\alpha<2 / 5$. Заметим, что функция $\widetilde{\psi}_{\varepsilon}$ является решением краевой задачи $(2.2)$ при $\lambda=\hat{\lambda}_{\varepsilon}, f=-\hat{f}_{\varepsilon}$. Тогда, воспользовавшись оценкой (2.16), получим (3.12). Лемма доказана.

Теорема 2.1 доказьвает первую часть утверждения теоремы 1.1 о сходимости собственного значения возмущенной задачи. Из леммы 3.1 следует, что $\lambda_{\varepsilon}=\hat{\lambda}_{\varepsilon}+o(\varepsilon)=$ $\lambda_{0}+\varepsilon \lambda_{1}+o(\varepsilon)$, где $\lambda_{1}$ определяется формулой (1.4). Таким образом, теорема 1.1 полностью доказана.

Автор выражает благодарность Р. Р. Гадыльшину за внимание к работе и полезные замечания.

\section{СПИСОК ЦИТИРОВАННОЙ ЛИТЕРАТУРЫ}

[1] Ландау Л. Д., Лифшиц Е. М. Квантовая механика. Нерелятивистская теория. М.: Наука, 1974.

[2] Ильин А. М. Согласование асимптотических разложений решений краевых задач. М.: Наука, 1989.

[3] Мазья В. Г., Назаров С. П., Пламеневский Б. А. Асимптотические разложения собственных чисел краевых задач для оператора Лапласа в областях с малыми отверстиями // Изв. АН СССР. Сер. матем. 1984. Т. 48. № 2. С. 347-371.

[4] Гадыльшин P. P. Асимптотика собственного значения сингулярно возмущенной эллиптической задачи с малым параметром в граничном условии // Дифференц. уравнения. 1986. T. 22. C. $640-652$.

[5] Гадыльшин Р. Р. Расщепление кратного собственного значения задачи Дирихле для оператора Лапласа при сингулярном возмущении граничного условия // Матем. заметки. 1992. Т. 52. № 4. С. $42-55$.

[6] Планида М. Ю. О сходимости решений сингулярно возмущенных краевых задач для лапласиана // Матем. заметки. 2002. Т. 71. №6. С. 867-877.

[7] Планида М. Ю. Об асимптотике собственных значений для цилиндра, теплоизолированного на узкой полосе // ЖВМиМФ. 2003. Т. 43. С. 422-432.

[8] Ладыженская О. А., Уральцева Н. Н. Линейные и квазилинейные уравнения эллиптического типа. М.: Наука, 1973.

[9] Михайлов В. П. Дифференциальные уравнения в частных производных. М.: Наука, 1976.

[10] Санчес-Паленсиа Е. Неоднородные среды и теория колебаний. М.: Мир, 1984.

Уфимский государственный авиационный технический университет E-mail: michmike@yandex.ru 